\title{
Portrait de donneurs de sang à Montréal (Canada) : typologie en fonction de leurs déplacements quotidiens
}

Marie-Soleil Cloutier, Élianne Carrier et Johanne Charbonneau

\section{(2) OpenEdition}

\section{Journals}

Édition électronique

URL : https://journals.openedition.org/rfst/1210

DOI : $10.4000 /$ rfst. 1210

ISSN : 2492-3672

Éditeur

Espaces et SOciétés (UMR 6590)

Référence électronique

Marie-Soleil Cloutier, Élianne Carrier et Johanne Charbonneau, «Portrait de donneurs de sang à Montréal (Canada) : typologie en fonction de leurs déplacements quotidiens », Revue francophone sur la santé et les territoires [En ligne], Miscellanées, mis en ligne le 16 juin 2021, consulté le 16 juin 2021. URL : http://journals.openedition.org/rfst/1210 ; DOI : https://doi.org/10.4000/rfst.1210

Ce document a été généré automatiquement le 16 juin 2021.

La Revue francophone sur la santé et les territoires est mise à disposition selon les termes de la Licence Creative Commons Attribution - Pas d'Utilisation Commerciale - Partage dans les Mêmes Conditions 4.0 International. 


\title{
Portrait de donneurs de sang à Montréal (Canada) : typologie en fonction de leurs déplacements quotidiens
}

\author{
Marie-Soleil Cloutier, Élianne Carrier et Johanne Charbonneau
}

\section{Introduction}

1 La mise sur pied de moyens efficaces pour trouver de nouveaux donneurs et pour les conserver est cruciale pour les agences responsables de l'approvisionnement en sang. Pour répondre à cette demande la recherche récente s'est orientée en particulier sur la compréhension des motivations individuelles associées au don de sang. Par exemple, des études qualitatives utilisant des questions ouvertes ont démontré que la pratique du don de sang est associée aux traits de personnalité (ex. : altruisme), aux influences sociales (ex.: donner avec sa famille), à la qualité du service aux donneurs par les employés des agences de don sur les lieux de collecte et aux mesures prises par les agences pour accommoder les donneurs (prise de rendez-vous, horaire des collectes, etc.) (Charbonneau, Cloutier, \& Carrier, 2015; Henrion, 2003; Szymanski, Cushna, Jackson, \& Szymanski, 1978). Par ailleurs, l'importance relative des motivations change au cours du temps, en fonction de l'implication des donneurs de sang (Piliavin \& Callero, 1991) et les motivations sont influencées par différents événements qui surviennent dans le parcours de vie du donneur (Charbonneau, Cloutier, \& Carrier, 2016; Piersma, Bekkers, de Kort, \& Merz, 2019). Par exemple, les mesures améliorant les aspects pratiques du don sont davantage une priorité lorsque les donneurs doivent conjuguer cette pratique avec des engagements professionnels et familiaux. En effet, dans les sociétés occidentales, la capacité à attirer de nouveaux donneurs et à les fidéliser est tributaire des aléas de la vie quotidienne et des horaires de plus en plus chargés des donneurs potentiels (Bianco, 2013; Charbonneau, Cloutier, \& Carrier, 2016; Godin et al., 2005). De fait, de récentes recherches sur les motivations des donneurs ont 
révélé que bon nombre d'entre eux réduisent ou cessent complètement leur participation lorsque leurs obligations personnelles - travail, famille, etc. deviennent trop prenantes (Bani, Strepparava, \& Giussani, 2014; Charbonneau et al., 2016; Hillgrove, Doherty, \& Moore, 2012; James et al., 2014; Piersma et al., 2019; Piersma, Bekkers, Klinkenberg, De Kort, \& Merz, 2017; Schreiber et al., 2006; Soliman \& Boenigk, 2019).

Devant l'ampleur du défi de la fidélisation et du renouvellement des donneurs et le faible nombre d'études sur le don de sang qui vont au-delà des motivations individuelles, il nous apparaît pertinent de questionner l'espace-temps du don. Cet article propose d'étudier les itinéraires quotidiens des donneurs afin de documenter le moment et le lieu où ce geste s'insère dans leur journée. Il sera ensuite possible de caractériser différents types de donneurs à partir de leur espace-temps le jour du don. Une meilleure compréhension de ces dynamiques spatio-temporelles apportera de nouvelles connaissances et sera certainement utile pour les agences responsables de l'approvisionnement en produits sanguins qui cherchent toujours à s'adapter aux pratiques et comportements de leurs donneurs.

\section{L'espace-temps du don de sang au quotidien : ce qu'on en sait}

L'accessibilité géographique au lieu de collecte demeure un facteur important. L'étude de Carrier (2013) a démontré différentes préférences quant aux lieux de collecte où les donneurs de sang se rendaient selon le type de milieu de vie où ils habitaient (rural, urbain et de banlieue). Ces préférences oscillaient entre les points de collectes mobiles de la municipalité/quartier, les lieux de travail et des cliniques dédiées (lieu de collecte fixe). Dans cette enquête, tous les répondants ont mentionné connaître d'autres lieux de collectes que ceux qu'ils fréquentent habituellement mais très peu étaient enclins à parcourir de grandes distances pour faire un don (Carrier, 2013). L'importance de la proximité géographique des lieux de collecte ressort aussi dans d'autres travaux internationaux étudiant l'expérience du don, en particulier du premier don. De fait, Gillepsie et ses collègues (2002) soulignent que l'installation d'un centre de collecte mobile près des lieux de travail ou des écoles est essentiel pour recruter de nouveaux donneurs. Un peu plus d'une décennie plus tard, Poon (2013) arrive à la même conclusion : les donneurs potentiels sont peu disposés à parcourir de longues distances pour se rendre à un centre de collecte. En ce sens, Poon (2013) souligne d'ailleurs que de futures recherches devraient évaluer les habitudes quotidiennes et les patrons de mobilité des donneurs potentiels afin d'établir à quels endroits et à quels moments les gens sont le plus susceptibles de faire un don de sang. Dans la même logique, un certain nombre de recherches indique qu'il est préférable que les agences misent sur des sites mobiles à des lieux stratégiques plutôt que sur des centres fixes (Carey et al., 2012; Harrington et al., 2007; James et al., 2014; Nagurney \& Dutta, 2019; Weidmann, Schneider, Litaker, Weck, \& Klüter, 2012). Selon les recherches menées par James et al. (2014), les sites mobiles sont d'ailleurs un bon moyen d'attirer un bassin de donneurs plus jeunes. C'est d'ailleurs la stratégie qui est privilégiée au Québec (Canada), où Héma-Québec, la société responsable de l'approvisionnement en sang pour toute la province, mise sur des collectes mobiles, où sont effectuées $73 \%$ des dons annuellement (Héma-Québec, 2019). 
4 Pour ce qui est de la temporalité du don, elle est surtout étudiée en lien avec les heures d'ouverture des centres de collecte, qu'ils soient fixes ou mobiles. À ce titre, les études démontrent que les organismes responsables ont tout intérêt à s'adapter aux horaires des donneurs plutôt que l'inverse (Klinkenberg, Romeijn, de Kort, \& Merz, 2018). Ainsi, si la population ciblée travaille la semaine durant la journée, le centre de collecte devrait demeurer ouvert en soirée et la fin de semaine (Yuan, Hoffman, Lu, Goldfinger, \& Ziman, 2011). À titre d'exemple, les recherches menées par Harrington et ses collègues (2007) ont démontré que les cliniques les plus efficaces étaient celles qui avaient des horaires atypiques, en dehors des heures de bureau régulières. D'autres études, dont celle effectuée par Ringwald et son équipe (2010), ont également permis d'établir que certains donneurs seraient plus enclins à continuer à donner du sang s'ils avaient la possibilité de prendre rendez-vous à l'avance. Dans le même sens, l'étude de Carrier (2013) soulève que l'attente sur les lieux de collectes apparaît comme étant l'inconvénient principal à donner du sang. Les répondants de cette étude affirment éviter certaines plages horaires afin de réduire les délais. Ainsi, la question du temps requis a été abordée dans quelques études et le manque de temps ressort alors comme une raison significative poussant les donneurs à réduire ou arrêter de donner (Carrier, Cloutier, \& Charbonneau, 2015; Charbonneau et al., 2016; Craig, Garbarino, Heger, \& Slonim, 2016). En somme, ces études, bien que peu nombreuses, démontrent que la temporalité du don se joue sur deux niveaux. En premier lieu au niveau de la distance géographique à parcourir : plus la distance est grande plus le temps requis augmente, et deuxièmement, au niveau du temps d'attente sur le lieu de collecte : moins de temps sur le site augmente l'expérience positive.

\section{Comment la géographie du temps peut-elle contribuer à optimiser l'approvisionnement en sang?}

5 Pour mieux comprendre comment s'insère le don de sang dans la vie quotidienne des donneurs, une approche combinant la géographie du temps et celle de la santé nous apparait à propos. Les individus se déplacent de façon quotidienne, voire plusieurs fois par jour, pour accomplir leurs activités et obligations. Ces déplacements dans l'espace constituent l'objet de recherche principal en géographie du temps, traduction de la time geography . Pour Hägerstrand (1970, 1985), le fondateur de cette branche disciplinaire de la géographie, l'espace-temps consiste en la dimension spatiale et temporelle des activités humaines qui se déroulent à un lieu précis à la fois, pour un temps déterminé, incluant le contexte et/ou le but de l'activité pratiquée (Ellegård, Hägerstrand, \& Lenntorp, 1977). Contrairement aux études portant seulement sur les lieux, la géographie du temps ajoute le fait que la chaine des déplacements entre les lieux ont aussi besoin d'être analysés pour mieux comprendre le phénomène à l'étude (Rainham, McDowell, Krewski, \& Sawada, 2010).

$6 \quad$ Le concept d'espace d'action, traduction du terme activity space a été utilisé par des géographes en complément à la théorie d'Hägerstrand (Golledge \& Stimson, 1997; Janelle \& Goodchild, 1983; Patterson \& Farber, 2015; Raine, 1978). Les espaces d'action se définissent comme «l'ensemble de lieux où s'observent les pratiques d'un individu » (Lord, Joerin, \& Thériault, 2009) et comportent un aspect spatial et temporel. Ainsi, l'espace d'action d'un individu représente le contact direct entre l'individu et son environnement (Lewin, 1951) et se définit à travers la distance (et les déplacements) et 
le temps. Les contours géographiques de l'espace d'action sont dessinés par les déplacements quotidiens (Golledge \& Stimson, 1997; Patterson \& Farber, 2015). Ceux-ci peuvent être : 1) des déplacements autour du domicile, sans autre destination que le retour à domicile; 2) des déplacements pour aller et revenir de ses activités quotidiennes (travail, achat, activité sociale, etc.); 3) des allers-retours autour d'activités ponctuelles (Golledge \& Stimson, 1997). L'aspect temporel de l'espace d'action concerne la fréquence (nombre de fois), la régularité (récurrent ou pas, par jour, semaine, mois) et la durée selon lesquelles un lieu est visité (Perchoux, Chaix, Cummins, \& Kestens, 2013), mais aussi selon le temps de trajet pour accéder aux lieux d'activités (F. Demoraes, Gouëset, \& Bouquet, 2019). Hägerstrand (1970) distingue les activités individuelles "fixes", temporellement et spatialement déterminées et difficilement interchangeables et celles «flexibles» qui au contraire peuvent facilement être planifiées à un autre moment ou lieu. Gärling et al. (1998) pour leur part parlent d'activités « habituelles », planifiées ou spontanées. Dans tous les cas, une activité peut être fixe spatialement et flexible temporellement et vice-versa et c'est ce qui caractérise les espaces d'action (Perchoux et al., 2013). Suivant cette logique, Golledge et Stimson (1997) soulignent quatre patrons spatio-temporels d'activités quotidiennes : 1) les activités planifiées régulièrement qui se produisent à un endroit spécifique et pour un temps prédéterminé, comme le travail ou l'école; 2) les déplacements pour se procurer des produits consommés fréquemment, qui reviennent régulièrement sans être fixes dans l'horaire, comme les emplettes alimentaires ; 3) les déplacements pour des activités spontanées qui offrent la possibilité d'y revenir dans un avenir rapproché selon l'expérience, ce que ces auteurs appellent les time-contagious activities, comme les lieux de diffusion culturelle ; et 4) les déplacements surprises, généralement occasionnés par une urgence ou un besoin à combler à court terme (Golledge \& Stimson, 1997). A partir de cette catégorisation, nous faisons l'hypothèse qu'il existe plusieurs profils de donneurs suivant la façon dont ils positionnent la pratique du don dans leur espace-temps quotidien et nous proposons d'examiner ces espace-temps dans le présent article.

7 Le concept d'espace d'action est multidisciplinaire et est fréquemment utilisé en planification urbaine, en géographie ou encore en marketing (Patterson \& Farber, 2015). Son application en santé publique est aussi répandue. L'espace d'action est alors utilisé afin de mesurer son influence sur diverses variables telles que : les différences sociales dans l'accès aux installations de santé (Arcury et al., 2005); le sentiment d'intégration à la communauté (Townley, Kloos, \& Wright, 2009); les inégalités en termes de santé mentale (Vallée, Cadot, Roustit, Parizot, \& Chauvin, 2011); la participation à des activités préventives de santé (Vallée, Cadot, Grillo, Parizot, \& Chauvin, 2010) ou des routines médicales (Mason, 2010); sur les comportements sexuels à risque (Vaughan, Kramer, Cooper, Rosenberg, \& Sullivan, 2017); l'exposition aux sources de nourriture et leur influence sur l'IMC (Kestens et al., 2012; Kestens, Lebel, Daniel, Thériault, \& Pampalon, 2010); le tabagisme (Shareck, Kestens, Vallée, Datta, \& Frohlich, 2016); le niveau d'activité physique (Smith, Foley, \& Panter, 2019); l'exposition à des contaminants atmosphériques (Setton et al., 2011). Bien que l'approvisionnement en sang relève d'une question de santé publique, il n'a pas encore été étudié sous l'angle des espaces d'action quotidiens des donneurs. De plus, le concept d'espace d'action ne semble pas avoir été étudié jusqu'à présent pour optimiser des comportements prosociaux/altruistes. Pourtant, le concept d'espace d'action pourrait mettre en lumière les contraintes rencontrées mais également les facteurs 
motivationnels au don des individus, à partir de leurs déplacements quotidiens le jour du don.

\section{Méthodes}

Cet article propose d'étudier les déplacements le jour du don afin de caractériser leur expérience de don dans leur quotidien. Pour ce faire, nous avons retracé en détails leurs destinations le jour de leur don à partir d'un entretien directif que nous avons effectué lors de leur visite à la collecte de sang, après leur propre don.

\section{Guide d'entretien}

9 Le guide d'entretien comportait trois sections. Tout d'abord une section sur le don en soi qui permettait de documenter (1) le type de don (sang total ou par aphérèse, c'est-àdire don de plaquette ou de plasma), (2) à quoi correspondait le lieu du don pour le donneur (lieu de travail, étude, loisirs, etc.) et (3) les raisons du choix de ce lieu (réponses libres). La deuxième section portait sur les caractéristiques sociodémographiques des donneurs, reconnues pour avoir une influence sur le don de sang et/ou les contraintes de temps: âge, sexe, scolarité, statut d'emploi (incluant l'horaire de travail), statut familial, incluant le nombre d'enfants à la maison, une variable importante dans les déplacements quotidiens (McDonald, 2008; McLaren, 2018; Pratt \& Hanson, 1991). Une dernière section servait à recueillir le plus d'informations possibles sur l'ensemble des lieux fréquentés le jour du don par les donneurs du point de départ du matin jusqu'au soir. La Figure 1 illustre un exemple du codage effectué à partir des propos recueillis lors de l'entretien: pour chacune des lieux visités, le donneur interrogé devait fournir la localisation selon une des trois options suivantes : nom du lieu si public; adresse; intersection de rues ou code postal. Le participant devait aussi, pour chacun de ces lieux, décrire le but de la visite et le temps approximatif qu'il y avait passé (cela inclut au lieu du don). Entre les destinations, le donneur devait fournir le mode de transport utilisé et la durée estimée du trajet. Le lieu de résidence se retrouvait ainsi au début et/ou à la fin du parcours de chacun des donneurs, mais il a seulement été retenu une fois pour ne pas dupliquer les points représentants ces lieux dans les analyses. 
Figure 1 : Codage des destinations et déplacements des donneurs le jour du don
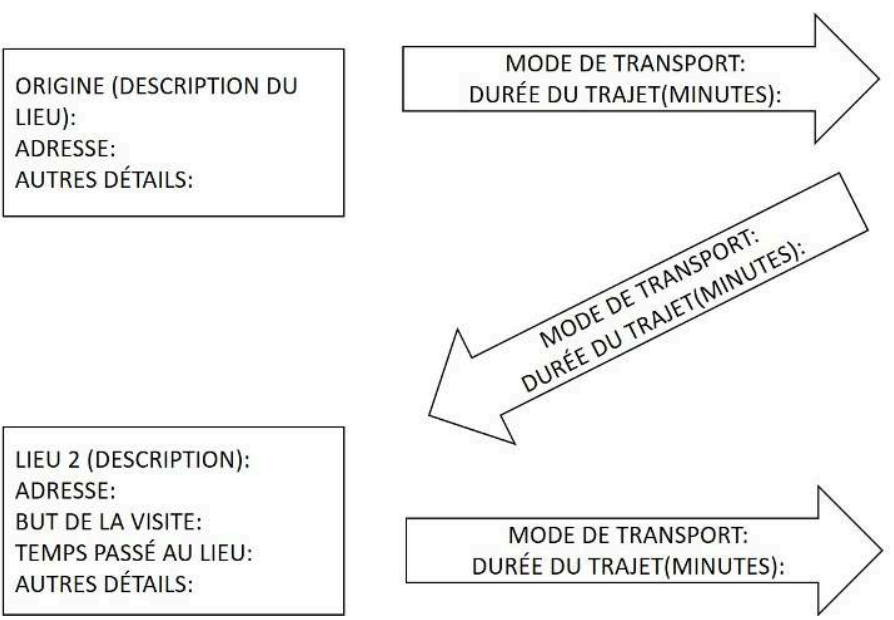

\section{Planification de la collecte de données sur les lieux de don de sang}

10 Ce projet a été fait en collaboration avec Héma-Québec et leur service de planification des collectes nous a fourni les informations et l'autorisation de se présenter tant sur des lieux de collectes fixes que mobiles choisis au hasard (via un tirage aléatoire dans leur base de données) dans la période visée pour notre projet. La région métropolitaine de Montréal, au Québec (Canada : voir Figure 2) a été choisi puisque c'est celle avec le plus de collecte dans la province. Pour ce qui est des dons sur rendez-vous aux collectes fixes, nous avons pu communiquer avec ces donneurs préalablement afin de les informer de notre étude et de les inviter à participer après leur don. Il est à noter que ce projet avait d'abord été approuvé par les comités d'éthique d'Héma-Québec et de l'INRS (numéro du certificat d'éthique : 14-356).

11 Dix-sept lieux de collecte ont été visités au total entre janvier et mars 2015 dans divers sites (centres commerciaux, écoles et universités, fêtes de quartier, etc. : voir Figure 2). Seulement deux de ces collectes étaient à des sites fixes puisque la quasi-totalité des collectes au Québec ont lieu à des sites mobiles (Héma-Québec, 2017). Les donneurs étaient approchés au moment du repos post-don pour répondre au questionnaire qui prenait entre 10 et 15 minutes, selon le nombre de destinations du donneur. Au total, 216 donneurs, dont 17 par aphérèse (plaquette ou plasma) ont participé, mais ces derniers ont été exclus des analyses en raison de leur profil particulier (Charbonneau, Cloutier, Carrier, \& Fainstein, 2017). Au final 199 questionnaires ont été analysés dans la présente étude. 
Figure 2 : Localisation des lieux de collecte visités dans la région métropolitaine de Montréal, Qc, Canada

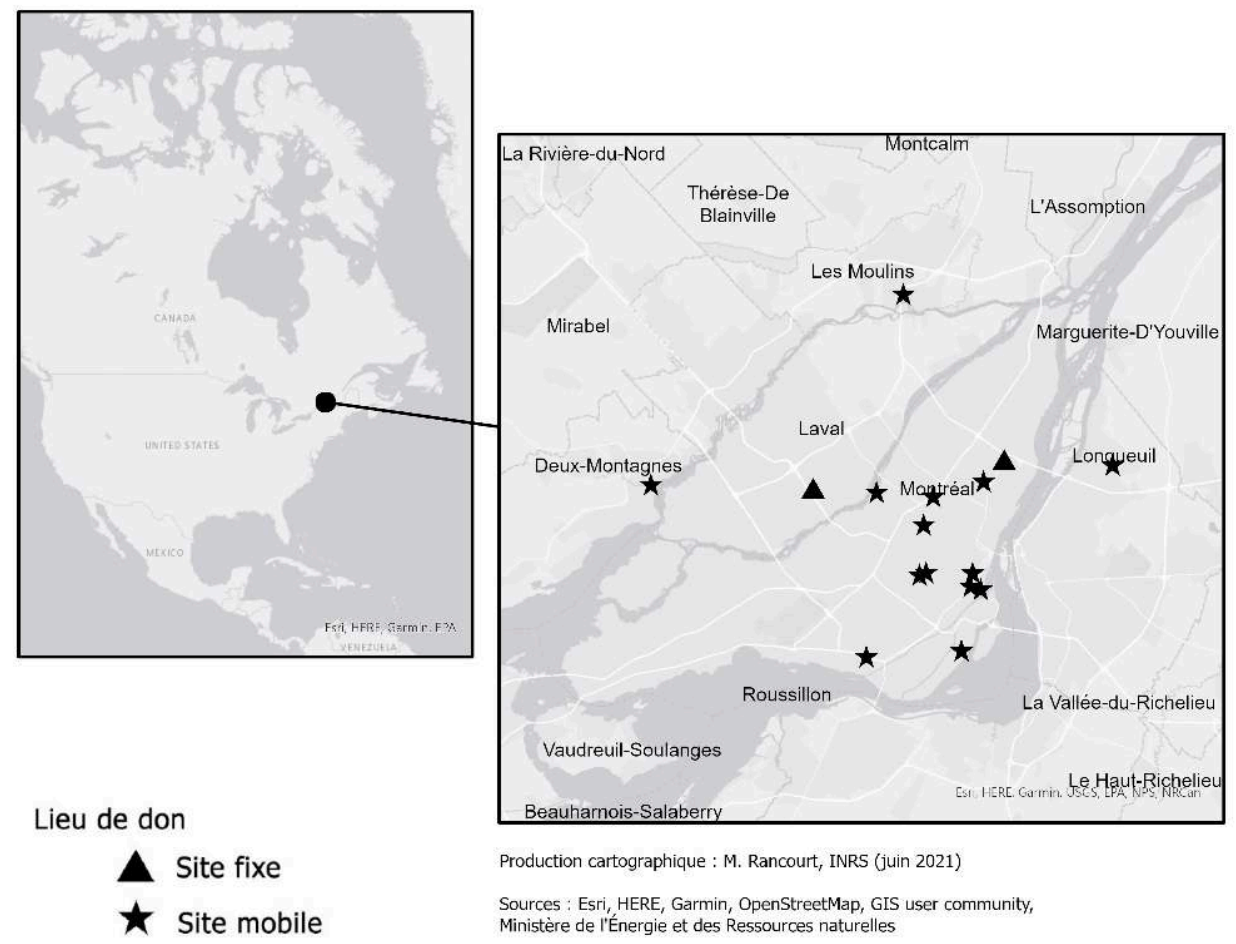

\section{Analyse spatiale des itinéraires : ellipse de distance à partir des destinations}

Toutes les destinations (lieux visités) et l'origine (domicile) ont été cartographiées à partir des informations fournies par le donneur lors de l'entretien. L'outil de géocodage du logiciel ArcGIS 10.3 (ESRI, 2017) et les couches d'informations spatiales de référence, à savoir le réseau routier d'Adresse-Québec (2016) et les codes postaux canadiens (Statistiques Canada, 2016) ont été utilisés pour cartographier tous ces lieux sous forme de points.

13 À partir de ces points, deux traitements d'analyse spatiale ont été effectués. Tout d'abord, les trajets sur le réseau routier ont été recréés en suivant la séquence des lieux visités pour chaque donneur. La reconstitution de ces trajets (via un algorithme du plus court chemin dans le module Network Analyst d'ArcGIS 10.3) nous a permis d'estimer les distances parcourues sur le réseau routier montréalais pour la journée du don pour chacun des répondants. Ce choix méthodologique du plus court chemin a été fait en raison du fait que nous n'avions pas d'information précise sur le chemin parcouru. Par ailleurs, cette méthode peut assurément sous-estimer les distances et les temps de transport, une limite qui est donc inhérente à nos résultats sur les distances parcourues, mais qui est présente de la même façon sur tout le territoire couvert.

Par la suite, des ellipses de distance standard ont été calculées pour les donneurs ayant au moins trois destinations différentes (le nombre minimal nécessaire pour la formation d'une ellipse) (Schönfelder \& Axhausen, 2004). La création d'ellipses de distance fait partie des méthodes propre à l'analyse centrographique et elles sont 
utilisées par des géographes dès les années 1920 pour visualiser la répartition d'un phénomène dans l'espace (Bachi, 1962; Lefever, 1926). Notre choix s'est porté sur cette méthode pour trois raisons. Tout d'abord, c'est celle choisie et éprouvée dans plusieurs écrits sur les espaces d'actions (Dijst, 1999; Horton \& Reynolds, 1971; Lord et al., 2009; Patterson \& Farber, 2015; Schönfelder \& Axhausen, 2003; Sherman, Spencer, Preisser, Gesler, \& Arcury, 2005). Deuxièmement, elle est assez simple à utiliser à partir des données que nous avons recueillies : elle utilise le semis de points (i.e. les destinations $\mathrm{du}$ donneur le jour du don, qui ne sont pas très nombreuses) pour dessiner une ellipse et ainsi calculer une "aire » d'activité, un indicateur que nous pouvons utiliser par la suite pour décrire le type de donneurs. Finalement, la possibilité de paramétrer la création des ellipses en donnant du poids aux lieux de collecte nous permet aussi de mieux visualiser les différents types d'espace d'activité. Malgré ces avantages, cette méthode a aussi ses limites. Elle est sensible aux valeurs extrêmes, comme nous allons le voir dans nos résultats. Par ailleurs, bien que ces ellipses résument les multiples lieux visités par les donneurs en un "nuage» simple, leur interprétation requiert la prudence, notamment en lien avec les points de destination sous-jacents, qui ne sont pas nécessairement distribués aléatoirement dans l'espace occupé par l'ellipse.

Le calcul des ellipses s'est fait dans ArcGIS 10.3 (ESRI, 2017). Pour que chaque ellipse contienne le lieu de collecte, nous avons attribué un poids de 3 aux lieux de collecte et un poids de 1 aux autres lieux de passage et l'ellipse a été dessinée à partir du point moyen, à savoir le point avec un $\mathrm{x}$ et un $\mathrm{y}$ qui représentent la moyenne des coordonnées de tous les points (seule option dans ArcGIS 10.3). Une fois les ellipses créées, leur superficie a été calculée (pour l'ajouter aux analyses subséquentes) et elles ont été cartographiées pour observer leur distribution géographique sur le territoire étudié. Le choix s'est porté sur des ellipses reposant sur la distance à vol d'oiseau plutôt que la distance de Manhattan puisque, à l'échelle spatiale d'une région comme celle étudiée, les ellipses résultantes sont peu influencées par le type de calcul des distances. De plus, les résultats préliminaires (par rapport aux calculs avec la distance de Manhattan) étaient plus à même de refléter l'espace couvert pour le comparer entre types de donneurs. Il est à noter que 22 donneurs n'avaient pas le nombre minimum de lieux visités pour calculer leur ellipse ( $n=3$, pour être en mesure de dessiner une forme circulaire avec une aire) et que 13 autres donneurs avaient des ellipses dont la surface était nulle puisque les lieux visités étaient trop linéaires (aller-retour entre des lieux sur une même ligne). Au total, ce sont donc 164 répondants qui ont été inclus dans les analyses subséquentes.

\section{Types de donneurs selon leur espace d'action le jour du don : utilisation des méthodes de classification}

C'est à partir d'une classification ascendante hiérarchique (CAH) que les donneurs ont été catégorisés en fonction de leurs variables individuelles (âge, genre), sociodémographiques (statut d'emploi, avec enfants à la maison) et géographiques (nombre de destinations, distance parcourue, aire de l'ellipse). Ce type de traitement statistique descriptif a pour objectif de regrouper dans une même classe les individus qui se ressemblent et de distinguer le plus possible les classes entre elles (Lebart, Piron, \& Morineau, 2006). Le calcul des regroupements s'est fait sur ces variables quantitatives 
et dichotomiques (pour le genre, le statut d'emploi et le fait d'avoir un enfant à la maison) avec la méthode de Ward et la distance euclidienne entre les individus.

\section{Résultats}

\section{Portrait des répondants}

Le Tableau 1 présente les principales caractéristiques sociodémographiques des donneurs interrogés ( $\mathrm{n}=199)$. Parmi eux, presque autant d'hommes $(46,7 \%)$ que de femmes $(53,3 \%)$ ont accepté de répondre aux questionnaires. Tous les groupes d'âges sont représentés, avec une proportion légèrement plus élevée chez les 50-64 ans, suivant ainsi l'âge des donneurs les plus fréquents (Charbonneau et al., 2015; Cloutier, Apparicio, Dube, Charbonneau, \& Delage, 2012). Par ailleurs, nos participants sont plus scolarisés que la population générale avec près de la moitié d'entre eux détenant un diplôme universitaire, notamment chez les femmes. Par ailleurs, plus de $80 \%$ des répondants sont encore actifs sur le marché du travail. Nos répondants sont à plus de $80 \%$ des actifs à temps plein ou partiel, incluant $22 \%$ d'étudiants. Seulement le tiers des répondants a des enfants qui vivent à la résidence familiale.

Tableau 1 : Caractéristiques démographiques des donneurs interrogés

\begin{tabular}{|c|c|c|c|c|c|c|c|}
\hline & \multicolumn{2}{|c|}{ Tous } & \multicolumn{2}{|c|}{ Femme } & \multicolumn{2}{|c|}{ Homme } \\
\hline & & $n$ & $\%$ & $n$ & $\%$ & $n$ & $\%$ \\
\hline & & 199 & & 106 & & 93 & \\
\hline \multirow{5}{*}{ Age } & $18-24$ ans & 40 & $20 \%$ & 22 & $21 \%$ & 18 & $19 \%$ \\
\hline & $25-34$ ans & 46 & $23 \%$ & 25 & $24 \%$ & 21 & $23 \%$ \\
\hline & $35-49$ ans & 50 & $25 \%$ & 26 & $25 \%$ & 24 & $26 \%$ \\
\hline & $50-64$ ans & 55 & $28 \%$ & 30 & $28 \%$ & 25 & $27 \%$ \\
\hline & 65 ans et plus & 8 & $4 \%$ & 3 & $3 \%$ & 5 & $5 \%$ \\
\hline \multirow{3}{*}{ Scolarité } & $\begin{array}{l}\text { Primaire et } \\
\text { secondaire }\end{array}$ & 41 & $21 \%$ & 15 & $14 \%$ & 26 & $28 \%$ \\
\hline & $\begin{array}{c}\text { Pré-universitaire } \\
\text { et technique } \\
\text { (Cégep) }\end{array}$ & 65 & $33 \%$ & 37 & $35 \%$ & 28 & $30 \%$ \\
\hline & $\begin{array}{c}\text { Université } \\
\text { (tous les cycles) }\end{array}$ & 93 & $47 \%$ & 54 & $51 \%$ & 39 & $42 \%$ \\
\hline \multirow{2}{*}{ Statut d'emploi } & $\begin{array}{c}\text { Inactif (au foyer, } \\
\text { en interruption, } \\
\text { au chômage ou } \\
\text { retraité) }\end{array}$ & 32 & $16 \%$ & 16 & $15 \%$ & 16 & $17 \%$ \\
\hline & $\begin{array}{l}\text { Actif (travailleur } \\
\text { ou étudiant, } \\
\text { temps plein ou } \\
\text { partiel) }\end{array}$ & 167 & $84 \%$ & 90 & $85 \%$ & 77 & $83 \%$ \\
\hline \multirow{2}{*}{$\begin{array}{c}\text { Avec enfant à } \\
\text { la maison }\end{array}$} & Non & 131 & $66 \%$ & 69 & $65 \%$ & 62 & $67 \%$ \\
\hline & Oui & 68 & $34 \%$ & 37 & $35 \%$ & 31 & $33 \%$ \\
\hline
\end{tabular}

\section{Croisement entre les lieux de don et différentes variables démographiques}

Le Tableau 2 présente les résultats des croisements entre les lieux de don et différentes variables sociodémographiques (\% en ligne). On peut y voir qu'une grande majorité (69\%) des lieux de collecte étaient près du domicile (35\%) ou du lieu de travail/d'étude (34\%) des donneurs. Viennent ensuite les lieux où les donneurs se sont déplacés 
spécialement pour leur don (17\%), les hommes étant plus présents en proportion dans cette catégorie ( $22 \%$ contre $13 \%$ pour les femmes). Pour ce qui est des lieux de dons sur le parcours (parfois non-planifiés), ils sont choisis en plus grand nombre par les jeunes ( $13 \%$ contre $6 \%$ pour tous les répondants), tandis qu'aucun des donneurs ayant des enfants à la maison n'a donné dans ce type de lieu. L'effet de la retraite se fait possiblement sentir chez les 50-64 ans et les plus de 65 ans, qui choisissent en plus grande proportion les lieux près d'une activité extra-professionnelle ( $13 \%$ des $50-64$ et des 65 ans et plus contre $8 \%$ pour tous les répondants). Cela se reflète aussi dans le statut d'emploi, avec près de la moitié des inactifs, qui n'incluent pas uniquement les retraités, qui ont donné près du domicile et le tiers qui ont fait un déplacement spécifique vers le lieu du don. Les donneurs ayant des niveaux de scolarité plus élevé (pré-universitaire et technique/professionnalisant [cégep] et université) donnent plus souvent à un lieu près du travail/des études contrairement aux autres qui ont fait un déplacement spécifique vers le lieu du don.

Tableau 2 : Lieux de collecte et variables sociodémographiques (\% du total en ligne)

\begin{tabular}{|c|c|c|c|c|c|c|c|}
\hline & & $\begin{array}{l}\text { Près du } \\
\text { domicile }\end{array}$ & $\begin{array}{l}\text { Près du } \\
\text { travail ou } \\
\text { lieu } \\
\text { d'étude }\end{array}$ & $\begin{array}{c}\text { Sur le } \\
\text { parcours }\end{array}$ & $\begin{array}{l}\text { Près d'une } \\
\text { activité }\end{array}$ & $\begin{array}{l}\text { Fait un long } \\
\text { déplacement } \\
\text { pour le don }\end{array}$ & Autres \\
\hline \multirow{2}{*}{ Total } & (n) & 69 & 67 & 12 & 15 & 34 & 2 \\
\hline & $(\%)$ & $35 \%$ & $34 \%$ & $6 \%$ & $7 \%$ & $17 \%$ & $1 \%$ \\
\hline \multirow{2}{*}{ Sexe } & Femme & $38 \%$ & $36 \%$ & $6 \%$ & $7 \%$ & $13 \%$ & $0 \%$ \\
\hline & Homme & $31 \%$ & $31 \%$ & $5 \%$ & $9 \%$ & $22 \%$ & $2 \%$ \\
\hline \multirow{5}{*}{ Âge } & $18-24$ ans & $35 \%$ & $40 \%$ & $12 \%$ & $8 \%$ & $5 \%$ & $0 \%$ \\
\hline & $25-34$ ans & $24 \%$ & $48 \%$ & $4 \%$ & $0 \%$ & $24 \%$ & $0 \%$ \\
\hline & $35-49$ ans & $36 \%$ & $32 \%$ & $2 \%$ & $8 \%$ & $20 \%$ & $2 \%$ \\
\hline & $50-64$ ans & $40 \%$ & $24 \%$ & $6 \%$ & $13 \%$ & $15 \%$ & $2 \%$ \\
\hline & 65 ans et plus & $50 \%$ & $0 \%$ & $0 \%$ & $13 \%$ & $37 \%$ & $0 \%$ \\
\hline \multirow{3}{*}{ Scolarité } & $\begin{array}{l}\text { Primaire et } \\
\text { secondaire }\end{array}$ & $39 \%$ & $12 \%$ & $7 \%$ & $12 \%$ & $25 \%$ & $5 \%$ \\
\hline & Cégep & $35 \%$ & $40 \%$ & $6 \%$ & $5 \%$ & $14 \%$ & $0 \%$ \\
\hline & $\begin{array}{c}\text { Université } \\
\text { (tous les cycles) }\end{array}$ & $32 \%$ & $39 \%$ & $5 \%$ & $8 \%$ & $16 \%$ & $0 \%$ \\
\hline \multirow{2}{*}{ Statut d'emploi } & Inactif & $47 \%$ & $3 \%$ & $0 \%$ & $16 \%$ & $31 \%$ & $3 \%$ \\
\hline & Actif & $32 \%$ & $40 \%$ & $7 \%$ & $6 \%$ & $14 \%$ & $1 \%$ \\
\hline \multirow{2}{*}{$\begin{array}{l}\text { Avec enfant à la } \\
\text { maison }\end{array}$} & Non & $34 \%$ & $31 \%$ & $9 \%$ & $6 \%$ & $19 \%$ & $1 \%$ \\
\hline & Oui & $35 \%$ & $38 \%$ & $0 \%$ & $10 \%$ & $15 \%$ & $2 \%$ \\
\hline
\end{tabular}

\section{Destinations des donneurs et insertion du don dans la succession d'activités quotidiennes}

Les donneurs interrogés avaient visité entre 3 et 19 lieux dans la journée (moyenne et médiane à 6 lieux), incluant celui du don pour un total de 1210 points à cartographier et à synthétiser à l'aide des ellipses. Ces destinations sont de tous ordres, les plus fréquentes étant les lieux de travail ou d'étude, les lieux de magasinage et de service courants (épicerie, banque, pharmacie, cinéma, coiffure, garderie) et les lieux de transit (pour le transport en commun en particulier : stationnement incitatif pour y laisser sa voiture et prendre le train vers le centre-ville, station de métro, etc.). Ce sont près des deux tiers des donneurs interrogés qui ont donné du sang pendant, avant ou après le travail/les études. De ce nombre, $31 \%$ ont donné du sang à un arrêt entre leur lieu de travail/d'étude et d'autres sorties vers des destinations diverses ; $20 \%$ ont donné lors 
d'une pause (incluant la pause du midi) pendant leur journée de travail/d'étude et près de $10 \%$ ont donné en se rendant à une seule autre destination, celle de la collecte de sang, avant ou après leur journée de travail/d'étude. Le dernier tiers des donneurs interrogés qui n'ont pas donné en lien avec leur lieu de travail/d'étude l'ont fait entre diverses sorties (la semaine ou la fin de semaine), et c'est seulement 7\% des participants qui ne sont sortis que pour faire leur don durant la journée du don.

\section{Typologie des donneurs selon leur espace d'action le jour du don}

Le dendrogramme de la classification des donneurs selon les sept variables retenues nous permet de choisir de créer cinq groupes de donneurs. Le Tableau 3 résume les valeurs moyennes ou encore les proportions des variables retenues dans la classification pour chacun des groupes tandis que le Tableau 4 présente la proportion de chaque groupe en fonction du lieu du don. Finalement, les cartes 1 à 5 illustrent les ellipses (et un symbole proportionnel à l'aire des ellipses positionné sur le centroïde de l'ellipse) pour les donneurs de chacun des groupes.

Tableau 3 : Variables retenues pour la classification par type de donneurs

\begin{tabular}{|c|c|c|c|c|c|c|}
\hline & $\begin{array}{l}\text { Groupe A } \\
\text { Les } \\
\text { donneurs de } \\
\text { proximité }\end{array}$ & $\begin{array}{l}\text { Groupe B } \\
\text { Les donneurs } \\
\text { types }\end{array}$ & $\begin{array}{l}\text { Groupe C } \\
\text { Les } \\
\text { donneurs de } \\
\text { voisinage }\end{array}$ & $\begin{array}{c}\text { Groupe D } \\
\text { Les donneurs } \\
\text { multi- } \\
\text { destinations }\end{array}$ & $\begin{array}{c}\text { Groupe E } \\
\text { Les } \\
\text { donneurs } \\
\text { grands } \\
\text { voyageurs } \\
\end{array}$ & TOTAL \\
\hline $\mathbf{N}$ & 16 & 57 & 9 & 79 & 5 & 166 \\
\hline $\begin{array}{c}\text { Âge } \\
\text { (moyenne) }\end{array}$ & 44 & 41 & 40 & 38 & 30 & 39,5 \\
\hline $\begin{array}{c}\text { Nombre de } \\
\text { destinations } \\
\text { (moyenne) } \\
\end{array}$ & 5,3 & 5,9 & 4,4 & 6,7 & 7,2 & 6,2 \\
\hline $\begin{array}{c}\text { Distance } \\
\text { parcourue } \\
\text { (moyenne km) }\end{array}$ & 8,9 & 23,4 & 22,6 & 55,9 & 165,5 & 41,7 \\
\hline $\begin{array}{l}\text { Aire de l'ellipse } \\
\text { (moyenne } \mathbf{k m}^{2} \text { ) }\end{array}$ & 0,8 & 6,5 & 0,2 & 58,6 & 875,4 & 56,6 \\
\hline Femmes (\%) & 69 & 51 & 56 & 49 & 80 & 53 \\
\hline $\begin{array}{c}\text { Enfant à la } \\
\text { maison } \\
\text { (\% oui) }\end{array}$ & 31 & 37 & 11 & 39 & 60 & 37 \\
\hline $\begin{array}{l}\text { En emploi } \\
\text { (\% oui) }\end{array}$ & 75 & 79 & 89 & 91 & 60 & 84 \\
\hline
\end{tabular}

21 Le groupe A regroupe 16 donneurs « locaux » qui ont parcouru en moyenne les plus courtes distances lors de la journée de leur don. Ce sont en plus grande proportion des femmes et les trois quarts sont en emploi. La carte 1 illustre bien le caractère très local de leurs déplacements durant la journée du don: les ellipses sont très petites et se localisent près des différents lieux de dons. Ce résultat est confirmé par le type de lieu où ces participants ont été interrogés. Plus de $80 \%$ des répondants de ce groupe ont associé la collecte à un lieu près de la maison ou du travail (Tableau 4). Le groupe $B$ ( $\mathrm{n}=57$ donneurs) représente quant à lui plus ou moins le donneur «type », le "profil moyen ", c'est-à-dire que les moyennes et proportions des variables sont presque 
toutes au même niveau que notre échantillon total, sauf pour les distances parcourues, qui sont moindres. Ces donneurs ont aussi été interrogés dans presque tous les lieux, incluant une proportion non-négligeable (16\%) qui a fait un déplacement relativement long uniquement pour effectuer un don. Sur la carte 2, on peut voir que les ellipses de ces donneurs se concentrent sur des mouvements pendulaires entre la maison, un $2^{\mathrm{e}}$ lieu (habituellement le travail) et au moins un $3^{\mathrm{e}}$ lieu, ce qui donne une forme plus arrondie aux ellipses. Le groupe $\mathrm{C}(\mathrm{n}=9)$ se caractérise par la plus petite moyenne pour le nombre de destinations et la plus faible proportion d'enfants à la maison. Ce sont aussi des donneurs en majorité en emploi ( $89 \%$ ) et ils parcourent d'assez petites distances. Nous pouvons les appeler les donneurs " de voisinage", avec plus de la moitié de ces donneurs qui ont identifié le lieu de collecte comme étant près de leur domicile. La carte 3 nous permet d'ajouter que ces donneurs ne semblent faire que des déplacements entre des lieux assez rapprochés et que le don s'insère bien souvent au centre de ces déplacements. Le groupe D rassemble le plus grand nombre de donneurs $(\mathrm{n}=79)$ et représente des professionnels ( $91 \%$ en emploi) relativement jeunes (moyenne de 38 ans) avec des enfants à la maison (40\% d'entre eux). Ces donneurs «multidestinations » parcourent de plus grandes distances avec de multiples destinations dans leur espace d'activité du jour du don ( $2^{\mathrm{e}}$ plus haute moyenne avec près de 7 destinations). Cela représente des chaines de déplacement complexes, ce qui se reflète sur la carte 4 où les ellipses sont pour la plupart allongées et se superposent sur tout le territoire. Cette configuration est reliée au fait que certaines destinations de ces donneurs se retrouvent dans les périphéries (ex: le domicile) et d'autres dans les quartiers centraux de l'île de Montréal (ex: le lieu de travail/d'étude). Cette multiplication des destinations ressort aussi dans le type de collectes qu'ils ont fréquentées, avec $22 \%$ de ces donneurs qui ont fait un déplacement exprès pour leur don et plus de $60 \%$ qui ont donné près du travail ou du domicile (Tableau 4). Finalement, le groupe $\mathrm{E}$ est constitué de seulement cinq donneurs, dont quatre femmes. Les femmes de ce groupe, que nous appelons les «grandes voyageuses» sont celles qui ont parcouru en moyenne les plus grandes distances et atteint le plus grand nombre de destinations. La carte 5 nous illustre l'ampleur des déplacements pour ces donneurs, mais elle met aussi en lumière le fait que ces donneurs sont possiblement des exceptions de par leur routine de transport le jour du don : deux donneurs ont fait un déplacement exprès pour le don et les autres ont donné près du travail ou du domicile. 
Tableau 4 : Types de donneurs et lieu de collecte (tel que décrit par les répondants)

\begin{tabular}{|c|c|c|c|c|c|c|c|}
\hline & & $\begin{array}{c}\text { Proche du } \\
\text { domicile } \\
N=63\end{array}$ & $\begin{array}{l}\text { Lieu de travail } \\
\text { ou étude } \\
\mathrm{N}=\mathbf{4 8}\end{array}$ & $\begin{array}{l}\text { Parcours } \\
\text { entre le } \\
\text { travail et le } \\
\text { domicile } \\
\mathrm{N}=11\end{array}$ & $\begin{array}{c}\text { Proche } \\
\text { d'activités } \\
\mathrm{N}=12\end{array}$ & $\begin{array}{l}\text { Déplacement } \\
\text { assez long } \\
\qquad \mathrm{N}=30\end{array}$ & $\begin{array}{c}\text { Autre } \\
\mathrm{N}=2\end{array}$ \\
\hline \multirow{2}{*}{$\begin{array}{c}\text { Groupe A } \\
\text { Les } \\
\text { donneurs de } \\
\text { proximité }\end{array}$} & $\mathrm{N}$ & 6 & 7 & 0 & 2 & 1 & 0 \\
\hline & $\begin{array}{l}\% \text { dans } \\
\text { groupe }\end{array}$ & $38 \%$ & $44 \%$ & $0 \%$ & $13 \%$ & $6 \%$ & $0 \%$ \\
\hline \multirow{2}{*}{$\begin{array}{c}\text { Groupe B } \\
\text { Les } \\
\text { donneurs } \\
\text { types }\end{array}$} & $\mathrm{N}$ & 23 & 14 & 6 & 5 & 9 & 0 \\
\hline & $\begin{array}{l}\% \text { dans } \\
\text { groupe }\end{array}$ & $40 \%$ & $25 \%$ & $11 \%$ & $9 \%$ & $16 \%$ & $0 \%$ \\
\hline \multirow{2}{*}{$\begin{array}{c}\text { Groupe C } \\
\text { Les } \\
\text { donneurs de } \\
\text { voisinage }\end{array}$} & $\mathrm{N}$ & 5 & 3 & 0 & 0 & 1 & 0 \\
\hline & $\begin{array}{l}\% \text { dans } \\
\text { groupe }\end{array}$ & $56 \%$ & $33 \%$ & $0 \%$ & $0 \%$ & $11 \%$ & $0 \%$ \\
\hline \multirow{2}{*}{$\begin{array}{c}\text { Groupe D } \\
\text { Les } \\
\text { donneurs } \\
\text { multi- } \\
\text { destinations }\end{array}$} & $\mathrm{N}$ & 28 & 22 & 5 & 5 & 17 & 2 \\
\hline & $\begin{array}{l}\% \text { dans } \\
\text { groupe }\end{array}$ & $35 \%$ & $28 \%$ & $6 \%$ & $6 \%$ & $22 \%$ & $3 \%$ \\
\hline \multirow{2}{*}{$\begin{array}{l}\text { Groupe E } \\
\text { Les } \\
\text { donneurs } \\
\text { grands } \\
\text { voyageurs }\end{array}$} & $\mathrm{N}$ & 1 & 2 & 0 & 0 & 2 & 0 \\
\hline & $\begin{array}{l}\% \text { dans } \\
\text { groupe }\end{array}$ & $20 \%$ & $40 \%$ & $0 \%$ & $0 \%$ & $40 \%$ & $0 \%$ \\
\hline
\end{tabular}

Carte 1 : Groupe A, les donneurs de proximité (travail, étude, domicile)

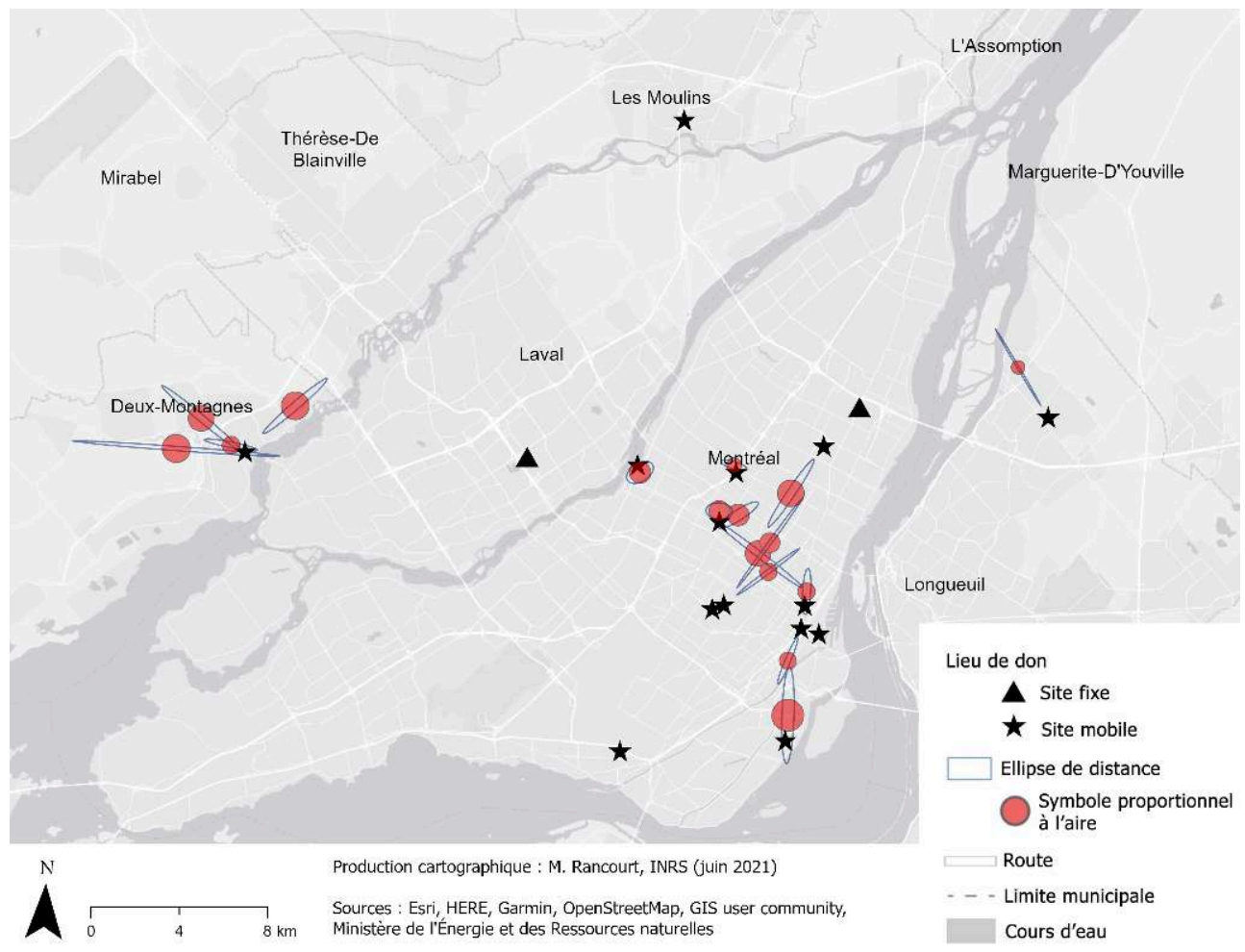


Carte 2 : Groupe B, les donneurs types

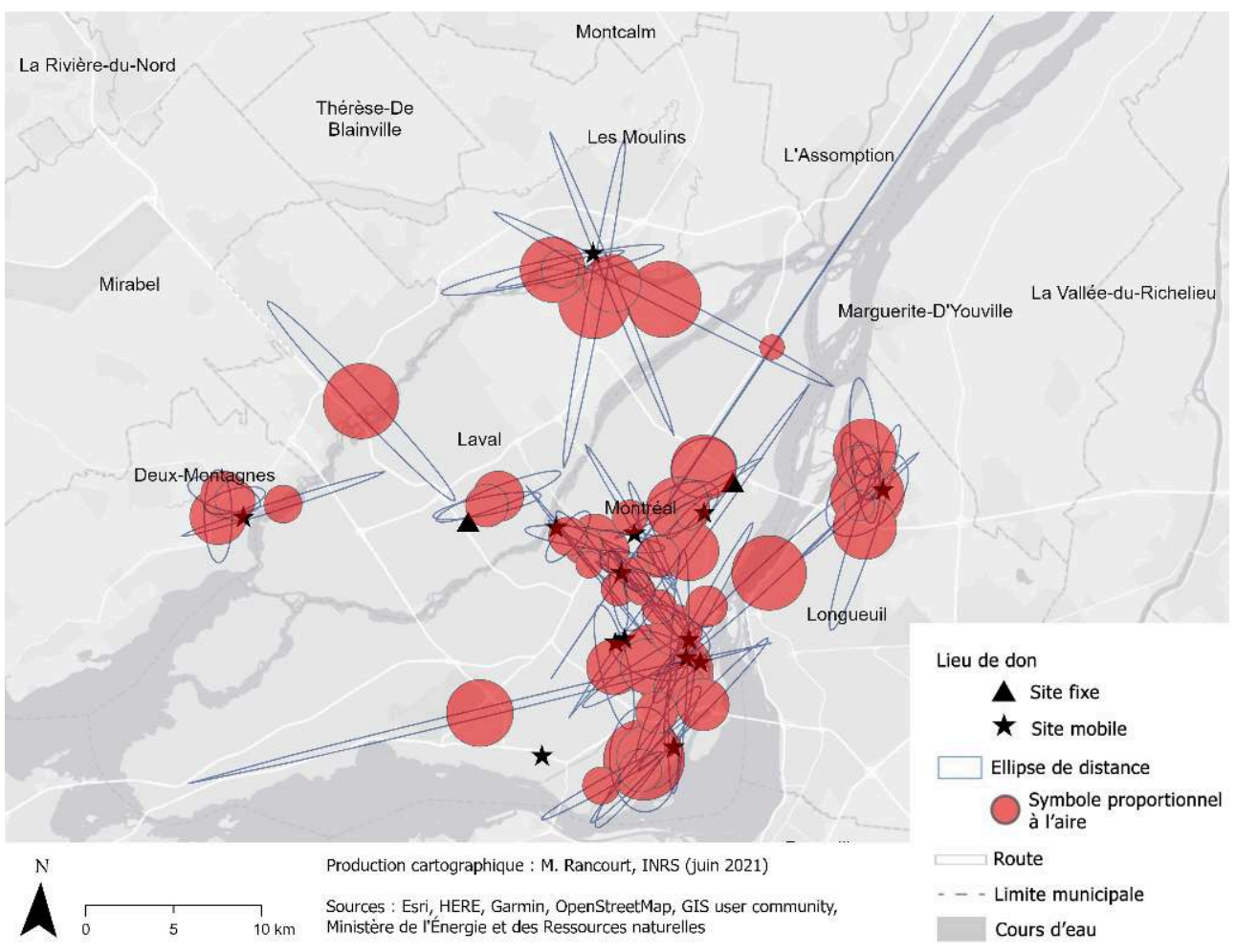

Carte 3 : Groupe $\mathrm{C}$, les donneurs de voisinage

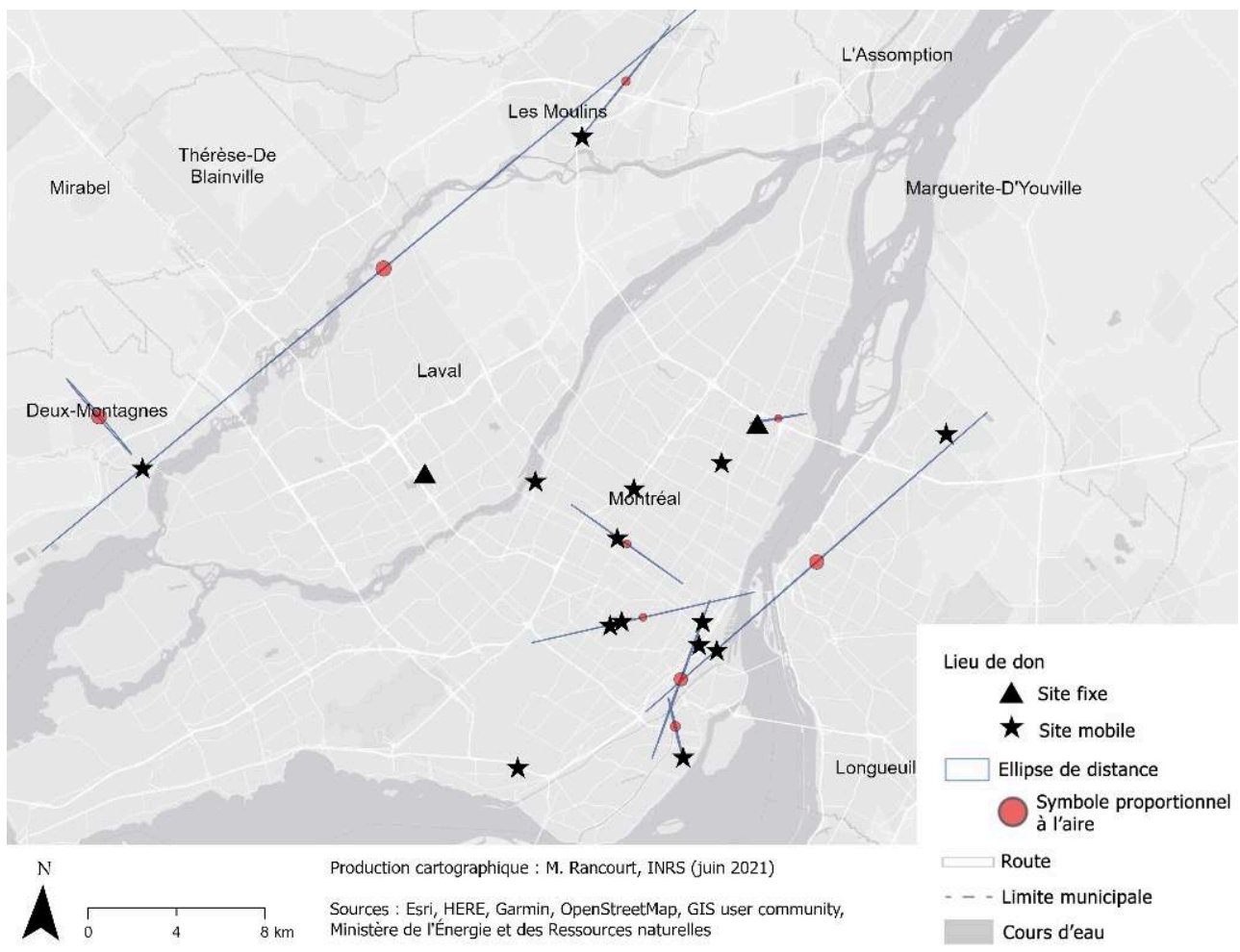


Carte 4 : Groupe D, les donneurs multi-destinations

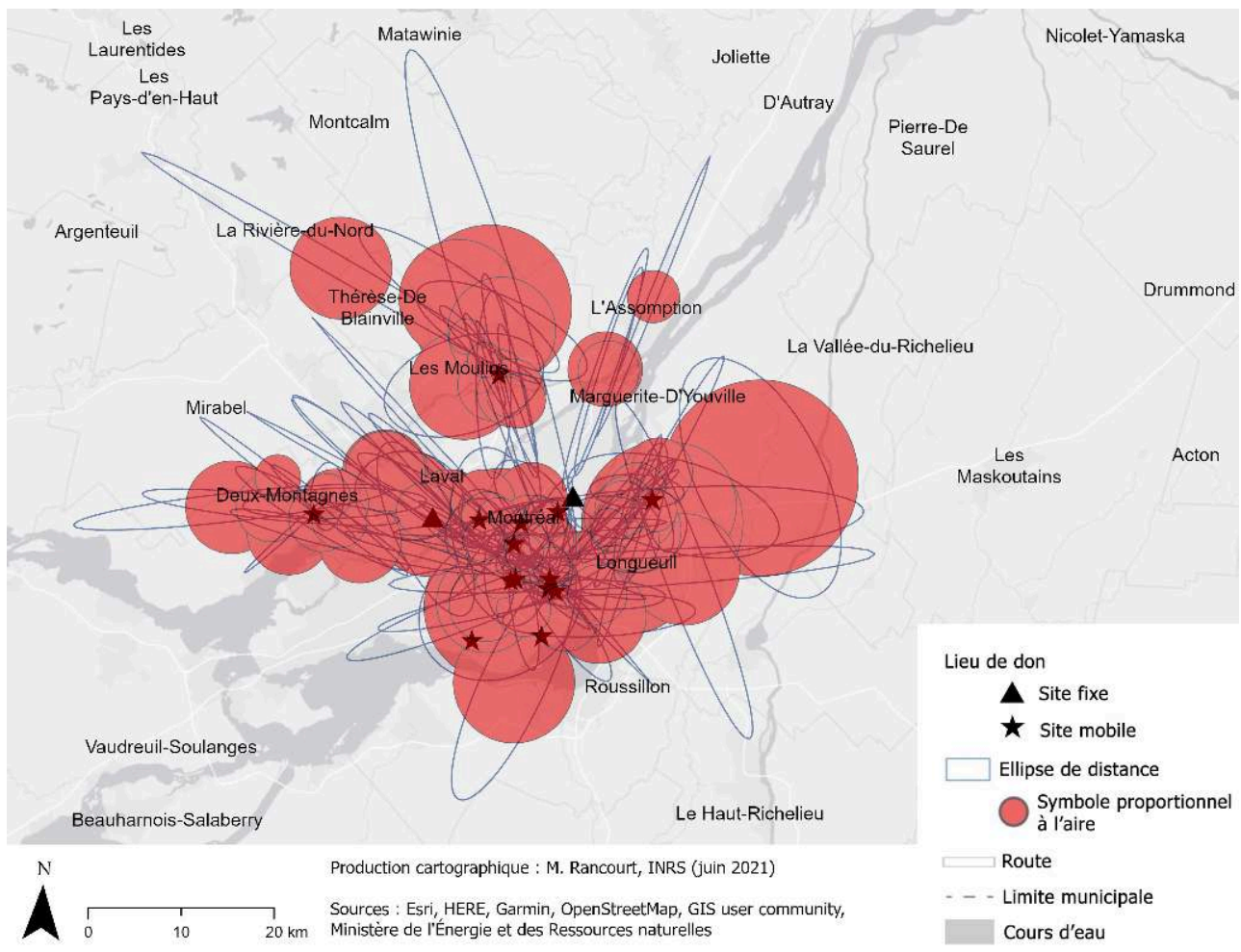

Carte 5 : Groupe E, les donneurs grands voyageurs

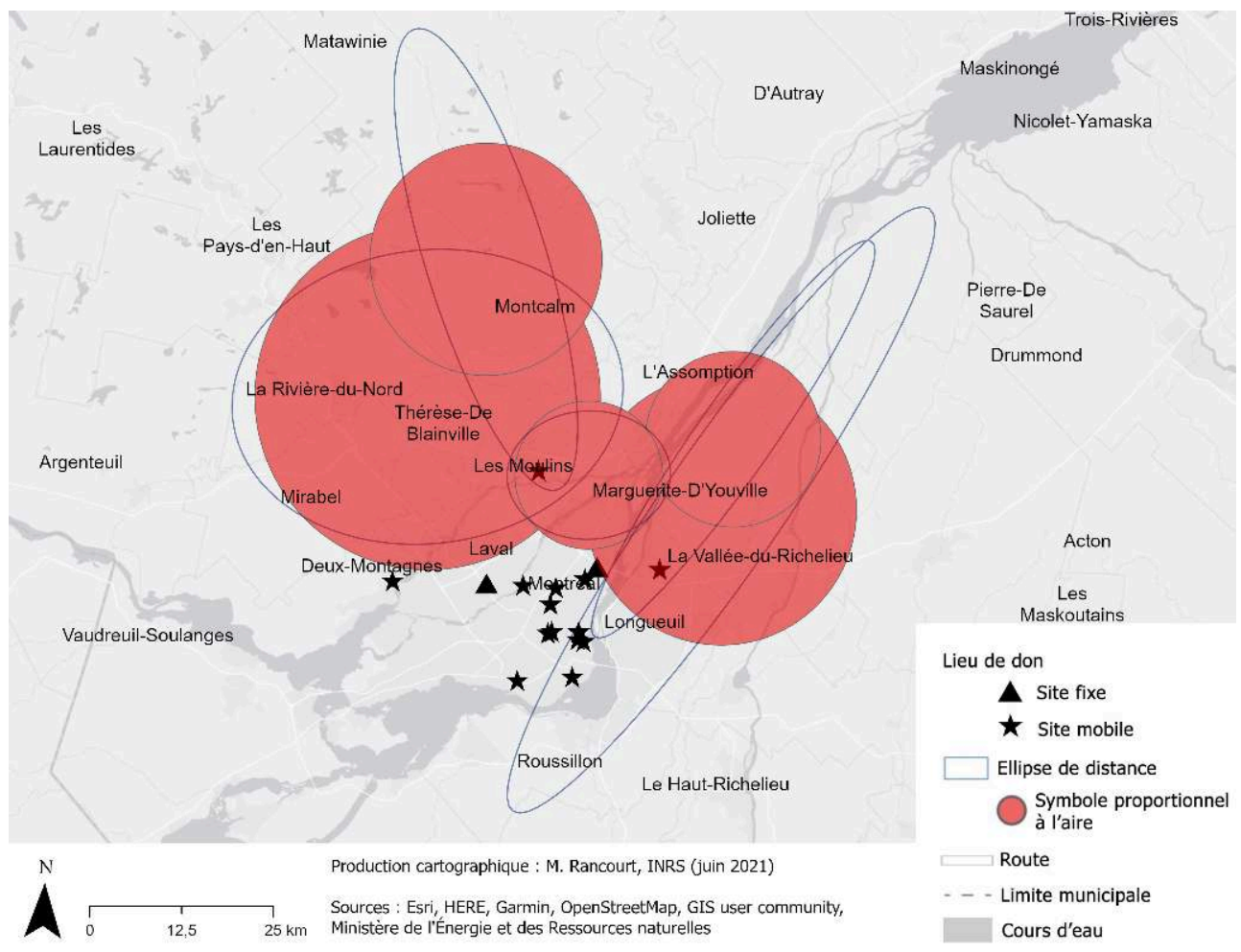




\section{Discussion}

22 La caractérisation des donneurs en fonction de leur espace d'action le jour de leur don nous en apprend plus sur l'insertion du don dans leur quotidien. L'hypothèse que nous avons émise au départ à savoir qu'il existe plusieurs types de donneurs avec des espaces d'action quotidiens différents les uns des autres s'avère exacte : nos résultats illustrent cinq types de donneurs provenant de la combinaison de leurs caractéristiques individuelles et de leurs itinéraires quotidiens. Notons tout de même que nos résultats ne sont pas sans limites inhérentes à la recherche. Tout d'abord, bien que nous ayons choisi les collectes à visiter aléatoirement, ce n'est pas tous les individus qui y étaient présents qui ont accepté de répondre à nos questions, ce qui représente un possible biais de sélection de l'échantillon qu'il est difficile de quantifier. Par ailleurs, le petit nombre de répondants sur la totalité des donneurs, ainsi que le fait que les lieux visités ont été cartographiés aussi précisément que ce que les participants nous ont rapportés (parfois à l'adresse exacte, parfois au code postal, moins précis) sont deux autres limites de ce projet. La relative complexité du guide d'entretien impliquait d'être présent avec le donneur pour bien colliger l'information, ce qui a limité le nombre de participants. Sans pouvoir généraliser les résultats à tous les donneurs, il nous apparait que ce projet vient tout de même éclairer une dimension encore inexplorée du don: son espace-temps. En ce sens, nous proposons ici deux constats qui sont autant de pistes de recherche et d'interventions futures pour les agences d'approvisionnement en sang.

Premièrement, la question de la proximité du lieu de don par rapport au domicile ou au lieu de travail/d'étude est très importante. Ce constat se retrouve dans les raisons justifiant le choix des lieux de don, mais aussi dans l'insertion du don sur l'itinéraire quotidien, entre des lieux phares (domicile/travail) et les autres destinations. Une question ouverte à la fin de l'entretien permettait aux participants de détailler les raisons de leur choix de ce lieu de don. Encore une fois, c'est la proximité à un autre lieu déjà fréquenté (centre commercial, lieu du travail ou des études, etc.) qui est ressorti comme étant le premier critère du choix de ce lieu de collecte. De même à la question s'ils devaient changer de lieu de collecte, celui-ci devrait être à proximité de lieux qu'ils fréquentent en cours de journée. Ce premier constat est en adéquation avec les études empiriques sur les espaces d'action, qui demeurent concentrés principalement autour du périmètre connu et habituel des gens (Zhang, Susilo, \& Ahmad Termida, 2016). De fait, les déplacements se jouent en fonction du domicile ou d'activités obligatoires comme aller travailler/étudier. Le poids des routines est élevé : les individus sont rarement amenés à déroger de leur trajet quotidien/habituel : ils vont faire des arrêts spontanés mais dans la majorité des cas ne prendront pas une route différente (Camarero \& Oliva, 2008; Golledge \& Stimson, 1997; Perchoux et al., 2014). Ce premier constat corrobore aussi ce qui est connu des écrits sur l'importance de la temporalité sur la motivation à donner. Ces écrits insistent sur le manque de temps comme une raison significative poussant les donneurs à réduire ou arrêter de donner (Carrier et al., 2015; Charbonneau et al., 2016; Craig et al., 2016). À la lumière de nos résultats, rapprocher les lieux de dons de ceux du quotidien (domicile et travail, entre autres) permettraient aux donneurs d'économiser du temps en réduisant, par exemple, leurs temps de transport, et d'ainsi réduire l'importance du manque de temps comme raisons de l'abandon du don. 
Deuxièmement, la dichotomie entre donneurs de milieu urbain, de banlieue ou de zones rurales, qui a été présentée à plusieurs reprises dans les travaux sur le don de sang, n'est pas présente dans cette étude (Carrier et al. 2015). Nous nous attendions à ce que les donneurs de milieux "rural » ou de "banlieue ", c'est-à-dire en dehors du centre de l'île de Montréal, aient des ellipses beaucoup plus dispersées que ceux du milieu « urbain». Au contraire, la forme, mais surtout la taille des ellipses dans les différents groupes de donneurs est similaire, peu importe où elles se retrouvent sur le territoire d'étude. En ce sens, nos résultats vont au-delà de cette dichotomie des milieux de vie "urbain» ou "de banlieue", mais illustre que les habitudes de déplacements quotidiens se catégorisent plutôt par les variables individuelles (âge, sexe, enfant à la maison) et les caractéristiques des trajets et le nombre de destinations. On retrouve ainsi des ellipses autant en ville qu'en banlieue dans chacun de nos groupes. Ces résultats sont aussi en opposition avec les travaux sur les espaces d'activités qui démontrent que les personnes en milieu rural ou de banlieue tendent à avoir des espaces d'action plus dispersés (polycentriques) (Hasanzadeh, 2019) alors que les citadins concentrent davantage leur action autour de leur lieu de travail et de leur domicile (monocentrique) (Buliung \& Kanaroglou, 2006; Camarero \& Oliva, 2008; Florent Demoraes, Piron, Zioni, \& Souchaud, 2012; Hasanzadeh et al., 2019; Lo \& Houston, 2018; Perchoux et al., 2014). Nos résultats suivent plutôt une logique de proximité à des lieux significatifs du quotidien, indépendamment du contexte résidentiel.

Comme nous en faisions l'hypothèse en introduction, l'utilisation des espaces d'action met en lumière certaines logiques spatio-temporelles au don des individus, à savoir la distance géographique aux lieux de don et leur proximité à d'autres lieux visités, ce qui ressort clairement de nos résultats, et ce, dans tous les milieux de vie. Nos deux constats originaux s'avèrent intéressants pour les agences d'approvisionnement en sang qui veulent augmenter leur bassin de donneurs potentiels en diversifiant leurs lieux de dons. Les collectes de sang se doivent d'être sur leur chemin du quotidien pour s'assurer d'attirer plus de donneurs de divers horizons : cibler les grands employeurs (au lieu de travail), les lieux générateurs de déplacement (stations de métro et de train) et divers lieux de rassemblement comme les centres commerciaux sont des pistes à explorer pour déployer des cliniques mobiles.

\section{Conclusions}

La relative rareté des recherches à ce jour sur l'insertion de la pratique du don de sang dans la vie quotidienne illustre tout de même le paradoxe entre les travaux sur les motivations qui démontrent l'importance des valeurs " morales » dans la décision du don et ceux sur les raisons de ne pas donner ou de l'abandon, qui se cristallisent alors autour de questions " pratiques ", de manque de temps, entre autres. Dans ce contexte, le présent projet de recherche avait pour objectif de documenter la routine d'un échantillon de donneurs le jour de leur don. À partir d'une enquête effectuée sur divers lieux de dons, il a été possible d'élaborer une typologie des donneurs en fonction de certaines variables individuelles et d'autres indicateurs reliés à leur mobilité quotidienne à partir de leur itinéraire.

C'est ainsi que nous avons pu constater que notre échantillon de donneurs reflétait une diversité des situations d'insertion du don dans l'itinéraire quotidien. Soulignons que 
les diverses mesures mises en place par Héma-Québec dans le déploiement de ses collectes mobiles (proximité de grands lieux d'emplois, présence dans les centres commerciaux et dans les centres communautaires, prise de rendez-vous et horaires flexibles) semblent répondre à des besoins tout aussi divers lorsque vient le temps de choisir un moment et un lieu pour donner du sang. À ce titre, cette agence d'approvisionnement en sang fait figure d'exemple pour sa capacité à rejoindre les donneurs où ils sont, quand ils sont disponibles.

\section{BIBLIOGRAPHIE}

Adresses Québec. (2016). AQ Réseau: Réseau routier et odonymes.

Arcury, T. A., Gesler, W. M., Preisser, J. S., Sherman, J., Spencer, J., \& Perin, J. (2005). The effects of geography and spatial behavior on health care utilization among the residents of a rural region. Health Services Research, 40 (1), 135-155. doi:10.1111/j.1475-6773.2005.00346.x

Bachi, R. (1962). Standard distance measures and related methods for spatial analysis. Papers in Regional Science, 10 (1), 83-132. doi: https://doi.org/10.1111/j.1435-5597.1962.tb00872.x

Bani, M., Strepparava, M., \& Giussani, B. (2014). Gender differences and frequency of whole blood donation in Italian donors: Even though I want to, I cannot? Transfusion and Apheresis Science, 50 (1), 81-86. doi:10.1016/j.transci.2013.11.001

Buliung, R. N., \& Kanaroglou, P. S. (2006). Urban Form and Household Activity-Travel Behavior. Growth and Change, 37 (2), 172-199. doi:10.1111/j.1468-2257.2006.00314.x

Camarero, L. A., \& Oliva, J. (2008). Exploring the Social Face of Urban Mobility: Daily Mobility as Part of the Social Structure in Spain. International Journal of Urban and Regional Research, 32 (2), 344-362. doi: https://doi.org/10.1111/j.1468-2427.2008.00778.x

Carey, P. M., High, P. M., Schlumpf, K. S., Johnson, B. R., Mast, A. E., Rios, J. A., . . for the, N. R. E. D. S., II. (2012). Donation return time at fixed and mobile donation sites. Transfusion, 52 (1), 127-133. doi:10.1111/j.1537-2995.2011.03235.x

Carrier, É. (2013). Ville, village, banlieue: quelles différences quand vient le temps de donner du sang? (Master). Université du Québec, Institut national de la recherche scientifique, Québec.

Carrier, É., Cloutier, M.-S., \& Charbonneau, J. (2015). Cities, villages, and suburbs: What sets them apart when it comes to giving blood? The Canadian Geographer / Le Géographe canadien, 59 (4), 447-460. doi:10.1111/cag.12219

Charbonneau, J., Cloutier, M.-S., \& Carrier, É. (2015). Motivational Differences between Whole Blood and Apheresis Donors in Quebec, Canada: A Questionnaire-Based Survey in a Voluntary Nonremunerated Context. Journal of blood transfusion, 2015 (568259). doi:10.1155/2015/568259

Charbonneau, J., Cloutier, M.-S., \& Carrier, É. (2016). Why Do Blood Donors Lapse or Reduce Their Donation's Frequency? Transfusion Medicine Reviews, 30 (1), 1-5. 
Charbonneau, J., Cloutier, M.-S., Carrier, É., \& Fainstein, B. (2017). Motivations et carrière des donneurs de plasma et de plaquettes au Québec . Retrieved from Montréal: http://espace.inrs.ca/id/ eprint/5767/1/charbonneau-2017-apherese.pdf

Cloutier, M.-S., Apparicio, P., Dube, J., Charbonneau, J., \& Delage, G. (2012). Regional variation in the modeling of donation frequency: the case of Hema-Quebec, Canada. Transfusion, 52 (11), 2329-2338. doi:10.1111/j.1537-2995.2012.03604.x

Craig, A. C., Garbarino, E., Heger, S. A., \& Slonim, R. (2016). Waiting To Give: Stated and Revealed Preferences. Management Science, 63 (11), 3672-3690. doi:10.1287/mnsc.2016.2504

Demoraes, F., Gouëset, V., \& Bouquet, M. (2019). Mesurer l'évolution de l'accessibilité à l'aide des "espaces-temps d'action" : l'exemple de Bogotá entre 1993 et 2009. Cybergeo. doi:10.4000/ cybergeo. 33638

Demoraes, F., Piron, M., Zioni, S., \& Souchaud, S. (2012). Inégalités d'accès aux ressources de la ville analysées à l'aide des mobilités quotidiennes : approche méthodologique exploratoire à São Paulo. Cahiers de géographie du Québec, 56 (158), 463-490. doi: https://doi.org/10.7202/1014555ar

Dijst, M. (1999). Action space as planning concept in spatial planning. Netherlands Journal of Housing and the Built Environment, 14 (2), 163-182. Retrieved from http://www.jstor.org/stable/ 41107791

Ellegård, K., Hägerstrand, T., \& Lenntorp, B. (1977). Activity Organization and the Generation of Daily Travel: Two Future Alternatives. Economic Geography, 53 (2), 126-152. doi:10.2307/142721

ESRI. (2017). ArcGIS 10.3. Redlands: Environmental Systems Research Institute.

Gärling, T., Gillholm, R., \& Gärling, A. (1998). Reintroducing attitude theory in travel behavior research: The validity of an interactive interview procedure to predict car use. Transportation, 25 (2), 129-146. doi:10.1023/A:1005004311776

Gillespie, T. W., \& Hillyer, C. D. (2002). Blood donors and factors impacting the blood donation decision. Transfusion Medicine Reviews, 16 (2), 115-130. doi:10.1053/tmrv.2002.31461

Golledge, R. G., \& Stimson, R. J. (1997). Spatial behavior: A geographic perspective . New York: Guilford Press.

Hägerstrand, T. (1970). What about people in regional science? Papers in Regional Science, 24 (1), 7-24. doi:10.1111/j.1435-5597.1970.tb01464.x

Hägerstrand, T. (1985). Time-geography: Focus on the Corporeality of Man, Society and Environment . Paper presented at the The Science and Praxis of Complexity, Montpellier, France.

Harrington, M., Sweeney, M. R., Bailie, K., Morris, K., Kennedy, A., Boilson, A., . . Staines, A. (2007). What would encourage blood donation in Ireland? Vox Sanguinis, 92 (4), 361-367. doi: 10.1111/j.1423-0410.2007.00893.x

Hasanzadeh, K. (2019). Exploring centricity of activity spaces: From measurement to the identification of personal and environmental factors. Travel Behaviour and Society, 14 , 57-65. doi: 10.1016/j.tbs.2018.10.001

Hasanzadeh, K., Czepkiewicz, M., Heinonen, J., Kyttä, M., Ala-Mantila, S., \& Ottelin, J. (2019). Beyond geometries of activity space: A holistic study of daily travel patterns, individual characteristics, and perceived wellbeing in Helsinki metropolitan area. Journal of Transport and Land Use, 12 (1), 149-177. doi:10.5198/jtlu.2019.1148

Héma-Québec. (2017). Rapport annuel 2016-2017 . Retrieved from Montréal: https://www.hemaquebec.qc.ca/userfiles/file/RA_2016-2017/RA_2016-2017_FR.pdf 
Héma-Québec. (2019). Rapport annuel 2018-2019 . Retrieved from Montréal: https://www.hemaquebec.qc.ca/userfiles/file/RA-2018-2019/Hema-Quebec_Rapport_annuel_2018-2019.pdf Henrion, A. (2003). L'énigme du don de sang. Approche ethnographique d'un don entre inconnus. (Master). Université de Liège,

Hillgrove, T. L., Doherty, K. V., \& Moore, V. M. (2012). Understanding non-return after a temporary deferral from giving blood: a qualitative study. BMC Public Health, 12 (1), 1063. doi: $10.1186 / 1471-2458-12-1063$

Horton, F. E., \& Reynolds, D. R. (1971). Effects of Urban Spatial Structure on Individual Behavior. Economic Geography, 47 (1), 36-48. doi:10.2307/143224

James, A. B., Josephson, C. D., Shaz, H., Schreiber, G. B., Hillyer, C. D., \& Roback, J. D. (2014). The value of area-based analyses of donation patterns for recruitment strategies. Transfusion, 54 (12), 3051-3060.

Janelle, D., \& Goodchild, M. (1983). Diurnal Patterns of Social Group Distributions in a Canadian City. Economic Geography, 59 (4), 403-425. doi:10.2307/144166

Kestens, Y., Lebel, A., Chaix, B., Clary, C., Daniel, M., Pampalon, R., . . p Subramanian, S. V. (2012). Association between Activity Space Exposure to Food Establishments and Individual Risk of Overweight. PLOS ONE, 7 (8), e41418. doi:10.1371/journal.pone.0041418

Kestens, Y., Lebel, A., Daniel, M., Thériault, M., \& Pampalon, R. (2010). Using experienced activity spaces to measure foodscape exposure. Health \& Place, 16 (6), 1094-1103. doi:10.1016/j.healthplace. 2010.06.016

Klinkenberg, E. F., Romeijn, B., de Kort, W. L., \& Merz, E. M. (2018). Reasons to end the donor career: a quantitative study among stopped blood donors in the Netherlands. Transfusion Medicine, 28 (3), 200-207. doi:10.1111/tme.12442

Lebart, L., Piron, M., \& Morineau, A. (2006). Statistique exploratoire multidimensionnelle: visualisations et inférences en fouille de données (4e éd. ed.). Paris: Dunod.

Lefever, D. W. (1926). Measuring Geographic Concentration by Means of the Standard Deviational Ellipse. American Journal of Sociology, 32 (1), 88-94. Retrieved from http://www.jstor.org/stable/ 2765249

Lewin, K. (1951). Field theory in social science: selected theoretical papers (D. Cartwright Ed.). New York: Tavistock publications LTD.

Lo, A. W. T., \& Houston, D. (2018). How do compact, accessible, and walkable communities promote gender equality in spatial behavior? Journal of Transport Geography, 68 , 42-54. doi:doi.org/10.1016/j.jtrangeo.2018.02.009

Lord, S., Joerin, F., \& Thériault, M. (2009). La mobilité quotidienne de banlieusards vieillissants et âgés: Déplacements, aspirations et significations de la mobilité. Canadian Geographer / Le Géographe canadien, 53 (3), 357-375. doi:10.1111/j.1541-0064.2009.00269.x

Mason, M. J. (2010). Attributing activity space as risky and safe: The social dimension to the meaning of place for urban adolescents. Health \& Place, 16 (5), 926-933. doi:10.1016/j.healthplace. 2010.05.004

McDonald, N. C. (2008). Household interactions and children's school travel: the effect of parental work patterns on walking and biking to school. Journal of Transport Geography, 16 (5), 324-331. doi: https://doi.org/10.1016/j.jtrangeo.2008.01.002 
McLaren, A. T. (2018). Parent-child mobility practices: revealing 'cracks' in the automobility system. Mobilities, 13 (6), 844-860. doi:10.1080/17450101.2018.1500103

Nagurney, A., \& Dutta, P. (2019). Competition for blood donations. Omega, 85 , 103-114. doi: 10.1016/j.omega.2018.06.001

Patterson, Z., \& Farber, S. (2015). Potential Path Areas and Activity Spaces in Application: A Review. Transport Reviews, 35 (6), 679-700. doi:10.1080/01441647.2015.1042944

Perchoux, C., Chaix, B., Cummins, S., \& Kestens, Y. (2013). Conceptualization and measurement of environmental exposure in epidemiology: Accounting for activity space related to daily mobility. Health \& Place, 21 , 86-93. doi:10.1016/j.healthplace.2013.01.005

Perchoux, C., Kestens, Y., Thomas, F., Hulst, A. V., Thierry, B., \& Chaix, B. (2014). Assessing patterns of spatial behavior in health studies: Their socio-demographic determinants and associations with transportation modes (the RECORD Cohort Study). Social Science \& Medicine, 119 , 64-73. doi:10.1016/j.socscimed.2014.07.026

Piersma, T. W., Bekkers, R., de Kort, W., \& Merz, E.-M. (2019). Blood Donation across the Life Course: The Influence of Life Events on Donor Lapse. Journal of Health and Social Behavior, 60 (2), 257-272. doi:10.1177/0022146519849893

Piersma, T. W., Bekkers, R., Klinkenberg, E. F., De Kort, W. L. A. M., \& Merz, E.-M. (2017). Individual, contextual and network characteristics of blood donors and non-donors: a systematic review of recent literature. Blood transfusion = Trasfusione del sangue, 15 (5), 382-397. doi: 10.2450/2017.0064-17

Piliavin, J. A., \& Callero, P. L. (1991). Giving blood: the development of an altruistic identity : Johns Hopkins University Press.

Poon, C. M., Lee, S. S., \& Lee, C. K. (2013). Variation of motivation between weekday and weekend donors and their association with distance from blood donation centres. Transfusion Medicine, 23 (3), 152-159. doi:10.1111/tme.12034

Pratt, G., \& Hanson, S. (1991). On the Links between Home and Work: Family-Household Strategies in a Buoyant Labour Market. International Journal of Urban and Regional Research, 15 (1), 55-74. doi: https://doi.org/10.1111/j.1468-2427.1991.tb00683.x

Raine, J. W. (1978). Summarizing Point Patterns with the Standard Deviational Ellipse. Area, 10 (5), 328-333. Retrieved from http://www.jstor.org/stable/20001388

Rainham, D., McDowell, I., Krewski, D., \& Sawada, M. (2010). Conceptualizing the healthscape: contributions of time geography, location technologies and spatial ecology to place and health research. Social Science \& Medicine, 70 (5), 668-676.

Ringwald, J. (2010). Established ways to keep donor's interest alive. ISBT Science Series, 5 (1), 17-23.

Schönfelder, S., \& Axhausen, K. W. (2003). Activity spaces: measures of social exclusion? Transport Policy, 10 (4), 273-286. doi:10.1016/j.tranpol.2003.07.002

Schönfelder, S., \& Axhausen, K. W. (2004). On the variability of human activity spaces. In M. KollSchretzenmayr, M. Keiner, \& G. Nussbaumer (Eds.), The real and virtual worlds of planning (pp. 237-262). Berlin: Springer.

Schreiber, G. B., Schlumpf, K. S., Glynn, S. A., Wright, D. J., Tu, Y., King, M. R., . . Guiltinan, A. M. (2006). Convenience, the bane of our existence, and other barriers to donating. Transfusion, 46 (4), 545-553. doi:10.1111/j.1537-2995.2006.00757.x 
Setton, E., Marshall, J. D., Brauer, M., Lundquist, K. R., Hystad, P., Keller, P., \& Cloutier-Fisher, D. (2011). The impact of daily mobility on exposure to traffic-related air pollution and health effect estimates. Journal of Exposure Science \& Environmental Epidemiology, 21 (1), 42-48. doi:10.1038/jes. 2010.14

Shareck, M., Kestens, Y., Vallée, J., Datta, G., \& Frohlich, K. L. (2016). The added value of accounting for activity space when examining the association between tobacco retailer availability and smoking among young adults. Tobacco Control, 25 (4), 406. doi:10.1136/ tobaccocontrol-2014-052194

Sherman, J. E., Spencer, J., Preisser, J. S., Gesler, W. M., \& Arcury, T. A. (2005). A suite of methods for representing activity space in a healthcare accessibility study. International Journal of Health Geographics, 4 (1), 24. doi:10.1186/1476-072x-4-24

Smith, L., Foley, L., \& Panter, J. (2019). Activity spaces in studies of the environment and physical activity: A review and synthesis of implications for causality. Health \& Place, 58 , 102113. doi: 10.1016/j.healthplace.2019.04.003

Soliman, M., \& Boenigk, S. (2019). Individual life events and blood giving. Journal of Consumer Marketing, 36 (7), 926-938. doi:10.1108/JCM-02-2018-2588

Statistiques Canada. (2016). Codes postaux canadiens .

Szymanski, L. S., Cushna, B., Jackson, B. C. H., \& Szymanski, I. O. (1978). Motivation of Plateletpheresis Donors. Transfusion, 18 (1), 64-68. doi:10.1046/j.1537-2995.1978.18178118567.x

Townley, G., Kloos, B., \& Wright, P. A. (2009). Understanding the experience of place: Expanding methods to conceptualize and measure community integration of persons with serious mental illness. Health \& Place, 15 (2), 520-531. doi:10.1016/j.healthplace.2008.08.011

Vallée, J., Cadot, E., Grillo, F., Parizot, I., \& Chauvin, P. (2010). The combined effects of activity space and neighbourhood of residence on participation in preventive health-care activities: The case of cervical screening in the Paris metropolitan area (France). Health \& Place, 16 (5), 838-852. doi:10.1016/j.healthplace.2010.04.009

Vallée, J., Cadot, E., Roustit, C., Parizot, I., \& Chauvin, P. (2011). The role of daily mobility in mental health inequalities: The interactive influence of activity space and neighbourhood of residence on depression. Social Science \& Medicine, 73 (8), 1133-1144. doi:10.1016/j.socscimed. 2011.08.009

Vaughan, A. S., Kramer, M. R., Cooper, H. L. F., Rosenberg, E. S., \& Sullivan, P. S. (2017). Activity spaces of men who have sex with men: An initial exploration of geographic variation in locations of routine, potential sexual risk, and prevention behaviors. Social Science \& Medicine, 175 , 1-10. doi: 10.1016/j.socscimed.2016.12.034

Weidmann, C., Schneider, S., Litaker, D., Weck, E., \& Klüter, H. (2012). A spatial regression analysis of German community characteristics associated with voluntary non-remunerated blood donor rates. Vox Sanguinis, 102 (1), 47-54. doi:10.1111/j.1423-0410.2011.01501.x

Yuan, S., Hoffman, M., Lu, Q., Goldfinger, D., \& Ziman, A. (2011). Motivating factors and deterrents for blood donation among donors at a university campus-based collection center. Transfusion, 51 (11), 2438-2444. doi:10.1111/j.1537-2995.2011.03174.X

Zhang, W., Susilo, Y. O., \& Ahmad Termida, N. (2016). Investigating the interactions between travellers' familiar areas and their multi-day activity locations. Journal of Transport Geography, 53 , 61-73. doi:10.1016/j.jtrangeo.2016.04.012 


\section{RÉSUMÉS}

Les agences d'approvisionnement en sang cherchent constamment des moyens de recruter et retenir les donneurs de sang. Comme peu d'études se sont penchées sur les aspects de la temporalité et des déplacements des donneurs de sang, l'objectif de cet article est d'étudier les itinéraires quotidiens de ces individus afin de documenter le moment et le lieu où ce geste s'insère dans leur journée et ainsi mieux cibler les interventions de recrutement et de rétention des donneurs. Les participants ont été rencontrés directement sur des lieux de collecte de sang $(n=199)$ où ils nous ont rapporté leur itinéraire du jour incluant la localisation des lieux qu'ils ont fréquentés. Deux traitements des données ont été fait à partir de ces données : nous avons créé des ellipses de distance, qui sont des zones géographiques formées à partir des points des lieux fréquentés au quotidien, et une classification ascendante hiérarchique a été élaborée à partir des variables individuelles et géographiques provenant des entretiens avec les donneurs. Les résultats de l'étude ont fait ressortir cinq types de donneurs qui se différencient selon leur âge, genre et statut familial et d'emploi, ainsi que selon leur parcours le jour du don (nombre et distance à leurs destinations, taille de l'ellipse). Les collectes à proximité soit du lieu de résidence ou de travail/étude restent privilégiées par la majorité $(n=136)$ des répondants mettant ainsi l'emphase sur les aspects « pratiques » entourant le geste de donner du sang, à savoir que les lieux de dons se doivent d'être insérés dans les trajets et routines quotidiennes pour avoir une certaine attractivité pour les donneurs.

\section{INDEX}

Mots-clés : Don de sang, mobilité quotidienne, espaces d'action, classification ascendante hiérarchique, ellipse de distance

Index géographique : Montréal

\section{AUTEURS}

\section{MARIE-SOLEIL CLOUTIER}

Professeure agrégée, Institut National de la Recherche Scientifique

\section{ÉLIANNE CARRIER}

Agente de recherche, Institut National de la Recherche Scientifique

JOHANNE CHARBONNEAU

Professeure retraitée, Institut National de la Recherche Scientifique 